\title{
Reliability applied to the Preventive Maintenance on Wind Farms using Weibull distribution with a Financial Approach
}

\author{
Leandro Ribeiro Alves da Silva and Ricardo Ferreira Pinheiro \\ Professional Master's in Electrical Energy \\ Universidade Federal do Rio Grande do Norte \\ Campus of Natal - Avenida Senador Salgado Filho, 3000 Natal (Brazil) \\ Phone/Fax number: +55 84 991846836, e-mail: leribeiro78@yahoo.com.br; ricpinh@ufrnet.br
}

\begin{abstract}
This article aims to describe the most adequate methodology in terms of period of time for the preventive maintenance items in a wind farm. The methodology is going to analyse parts of wind turbines that present constant events of corrective maintenance and determine the best period for a preventive action in order to optimize maintenance costs and maintenance time that is directly linked to the wind farm owner profit. The components that present more unpredictable failures will be recommended to an improved schedule of preventive maintenance based on historical record analysis applied to probability methods, bringing positive financial results in terms of maintenance and energy generation.
\end{abstract}

\section{Key words}

Reliability, Maintenance, Wind Turbines, Wind Farms, Weibull

\section{Introduction}

Reliability is the capacity of any component to deliver its required function, under operational conditions through a period of time [1]. Although the Reliability started to be studied with the main idea of only identifying defects in large scale manufacturing, recently Reliability has been applied in the projects implementation, in the plants maintenance [2] and in several other areas, including medical, insurance, etc. Such advances have driven the Reliability application to an improvement on the components availability, followed by companies profit increases or even just avoiding issues such as penalties related the lack of delivering energy into the contracts that is the case of the Wind Farms.

Reliability can be applicable to a maintenance reality once it is expected that the components will have their lifecycle well defined, decreasing the probability of unexpected failures. The confirmation of the Reliability calculations will bring several benefits, not only in the tangible aspects but also in the intangible aspects.

Currently, several good quality software options are available in the market, but most of them are not considering the profit losses due to the unscheduled maintenance based on the lack of generation.

Due to the important contribution that an appropriate maintenance Reliability can bring to an asset, a Reliability study in a Wind Farm is the aim of this article, in which a real situation will be presented followed by the calculation of the best scenario for the scheduled maintenance. After calculating an improved scheduled maintenance is expected that the plant availability, the profit losses and unscheduled maintenances record will be improved.

This article presents the scenario for improving the maintenance schedule by analysing the Reliability of some components in the Wind turbines. Most of the Brazilian's Wind Farms are hiring the turbine's manufacturer to operate and maintain their wind turbines. The Operations and Maintenance (O\&M) contracts are mostly time based, in which a Wind Farm availability target must be delivered by the Supplier. In the most recent contracts, a generation target based on the wind resource that achieves the Wind Farm has been agreed between the parties. On the two different kinds of contracts, the Reliability study is more than adequate to bring profits for both sides, the Supplier and the Owner, once the contracts set some clauses in which the extra generation by improving the availability or the generation is shared between both contract parties. Also, by generating more energy, the Owner protects himself against penalties under the Power Purchase Agreement (PPA). The article presents along the next sections the methodology applied followed by a case study, its results and the conclusions achieved.

\section{Description of Methodology}

The methodology to be applied in this article separates the analysed Wind Turbines in sectors [3] where each of the sectors present the component that fails more and also considering that a remote solution is not available for such cases. 
The components are then selected, simply by statistics and then the reliability study based on probability of occurrences will be put in place [4]. The selection process was made through a set of one year data from a Wind Farm in Brazil with 60 units of $800 \mathrm{~kW}$ Wind Turbines. The one year data was determined for this specific article, being possible to add as much data as possible in order to obtain a more accurate result.

The data was downloaded from the Wind Turbine record system and converted into a table format. For the selection criteria, the period of time that the turbine was stopped or in maintenance and the quantity of events for each selected component was taken in consideration.

This article is part of a scope of work in a bigger work in which a more detailed analysis will be performed in more components of a wind turbine in order to mitigate unavailability and profit losses.

The article presents the solution found by the calculations to determine the best interval for the scheduled maintenances of the chosen component, taking into consideration the risk of failure [5] and the loss of electricity generation based on the annual energy production average for the wind farm. The calculation will adopt the Reliability function from the Weibull distribution.

The Weibull distribution that was described by the Swedish Wallodi Weibull on 1951 is extensively used to analyse lifetime data and consists on determining the scale and shape parameters plotting scenarios of reliability and probability [6].

The Probability $(\mathrm{F}(\mathrm{t}))$ and the Reliability $(\mathrm{R}(\mathrm{t}))$ functions for the Weibull distribution are shown below in the equations 1 and 2, respectively:

$$
\begin{aligned}
& \mathrm{F}(\mathrm{t})=1-\mathrm{e}^{-\left(\frac{\mathrm{t}}{\alpha}\right)^{\beta}} \\
& \mathrm{R}(\mathrm{t})=1-\mathrm{F}(\mathrm{t})
\end{aligned}
$$

Where:

$$
\begin{aligned}
& \mathrm{t} \text { - time to fail } \\
& \alpha \text {-scale parameter (based on hours) } \\
& \beta \text {-shape parameter (dimensionless) }
\end{aligned}
$$

After 1 year of data analysis, the component of the Wind Turbine that had shown more events of failure without the possibility of a remote restart was the fan system in the power cabinets. This fan system is responsible to maintain the power cabinets in a workable temperature, avoiding damages that can be caused by excess of heating in the Wind Turbine power system. Due to the importance of this system, it is not allowed to keep the turbines working.

\section{Case study}

Assessing the number of fails in the fan system in the power cabinet for a Wind Farm during 1 year, of an amount of 60 turbines, 10 events could not be remotely solved creating an outage in the turbines until the fan system replacement/maintenance could be done by the maintenance team. Most of the times, this kind of event led the wind turbine to stay out of work for more than 16 hours and it has created big losses in terms of energy production and availability results. The events had the following time characteristics shown in the following table:

Table I. Events of failure in the fan system in the power cabinets

\begin{tabular}{|c|c|}
\hline $\begin{array}{c}\text { Number of events in the } \\
\text { Power fan system }\end{array}$ & MTTF(h) \\
\hline 1 & 7200 \\
\hline 2 & 6480 \\
\hline 3 & 4320 \\
\hline 4 & 10080 \\
\hline 5 & 9360 \\
\hline 6 & 7920 \\
\hline 7 & 4320 \\
\hline 8 & 5760 \\
\hline 9 & 6480 \\
\hline 10 & 8640 \\
\hline
\end{tabular}

Calculating the time average for the events, the mean time to failure (MTTF) is of 7.056 hours. So, the average lifetime for performing maintenance or replacing completely the fan system should be at least before the 7.056 hours, but this practice does not bring the best result in terms of maintenance efficiency or risk mitigation, mostly due to five events of failures happening before the 7.000 hours of work in the sample analysed. Using the MTTF for the events it is possible to expand the calculations to a Reliability based on the Weibull distribution. The calculation as per Weibull analysis must find the scale and the shape parameters in order to know the best scenario for the scheduled maintenance based on the maintenance total cost, risk of failure and less losses of energy production.

By using some calculations in Microsoft Excel such as Median Rank, Linear Regression and the Weibull Distribution Function [7] it is possible to obtain the scale and shape parameters as below:

\begin{tabular}{|c|c|c|c|c|c|}
\hline MTTF & $\begin{array}{c}\text { Event } \\
\text { number }\end{array}$ & Median Rank & 1/(1-Median Rank) & $\operatorname{Ln}($ Ln(1/(1-Median Rank)) $)$ & Ln(MTTF) \\
\hline 4320 & 1 & 0,067307692 & 1,072164948 & $-2,663843085$ & 8,371011 \\
\hline 4320 & 2 & 0,163461538 & 1,195402299 & $-1,72326315$ & 8,371011 \\
\hline 5760 & 3 & 0,259615385 & 1,350649351 & $-1,202023115$ & 8,658693 \\
\hline 6480 & 4 & 0,355769231 & 1,552238806 & $-0,821666515$ & 8,776476 \\
\hline 6480 & 5 & 0,451923077 & 1,824561404 & $-0,508595394$ & 8,776476 \\
\hline 7200 & 6 & 0,548076923 & 2,212765957 & $-0,230365445$ & 8,881836 \\
\hline 7920 & 7 & 0,644230769 & 2,810810811 & 0,032924962 & 8,977146 \\
\hline 8640 & 8 & 0,740384615 & 3,851851852 & 0,299032932 & 9,064158 \\
\hline 9360 & 9 & 0,836538462 & 6,117647059 & 0,593977217 & 9,144201 \\
\hline 10080 & 10 & 0,932692308 & 14,85714286 & 0,992688929 & 9,218309 \\
\hline
\end{tabular}

Fig. 1. Median Rank Calculation into the Microsoft Excel

The third column can be calculated by the equations 3,4 , 5 and 6 :

$$
\text { Column number } 3=\frac{\mathrm{MTTF}-0,3}{\text { total number of events }+0,4}
$$

Forth column by the equation 4 : 


$$
\text { Column number } 4=\frac{1}{1-\left(\frac{\mathrm{MTTF}-0,3}{\text { total number of events }+0,4}\right)}
$$

Fifth column as in equation 5:

Column number $5=\operatorname{Ln}\left(\operatorname{Ln}\left(\frac{1}{1-\left(\frac{\text { MTTF }-0,3}{\text { total number of events }+0,4}\right)}\right)\right)(5)$

and the sixth column presented in the equation 6:

$$
\text { Column number } 6=\operatorname{Ln}(\mathrm{MTTF})
$$

In order to obtain the linear regression in the Microsoft Excel it is necessary to select as the input for the $\mathrm{Y}$ range the results in the column 5 and the input for the $X$ range the results in the column 6. Then, set the Microsoft Excel to generate the results in a new worksheet. Once the results are shown, the shape parameter result, can be immediately identified as $\operatorname{Ln}(\mathrm{MTTF})$. The result can be found in the cell B18 on the new created worksheet. In order to obtain the scale parameter the equation 7 must be applied:

$$
\text { Scale parameter equation }=\operatorname{EXP}\left(\frac{-\mathrm{B} 17}{\mathrm{~B} 18}\right)
$$

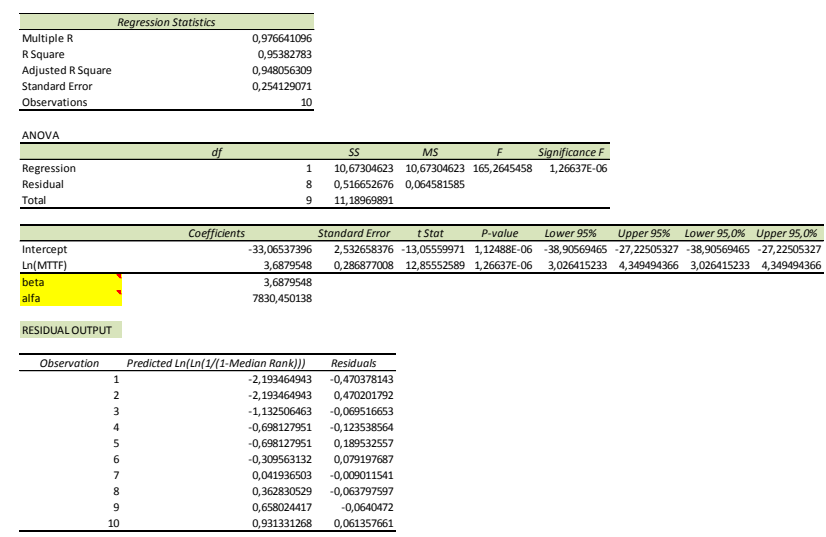

Fig. 2. Linear regression calculated in the Microsoft Excel

After calculating the scale parameter becomes easier to understand based on the bathtub curve below that the component that is being analysed is failing for exceeding its lifecycle. In the case of the shape parameter being smaller than 1, it means an early life failure, if it is equal to 1 means a constant level of failure and in case it is bigger than 1 means that the failure is going to happen due to the long lifetime of the component [8]. The shape parameter can be well represented by the bathtub curve below.

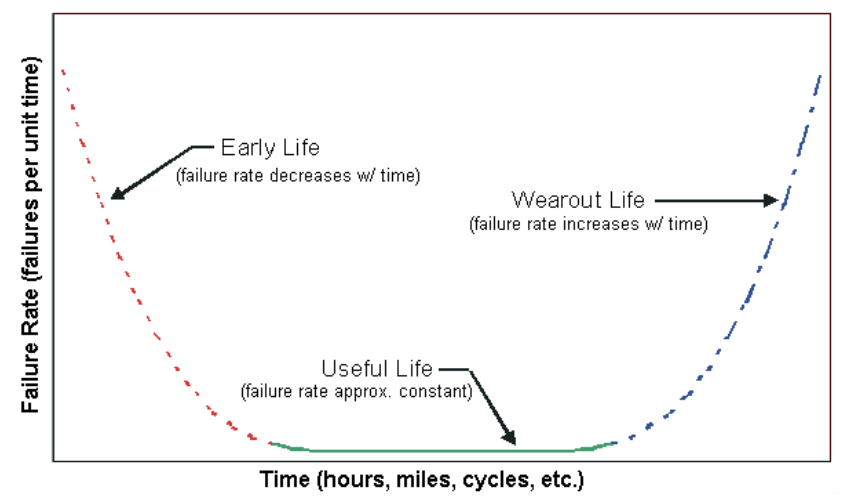

Fig. 3. Plot of a typical bathtub graph

\section{Results}

For the situation that is being presented in this article, the values for the scale and shape parameters are $7830 \mathrm{~h}$ and 3,6 respectively.

In order to proceed with the study is necessary to calculate the maintenance costs. Some assumptions were made such as in case of maintenance occur the system is considered as a new one. The schedule and unscheduled maintenance costs are being considered different only by the inclusion of the profit losses associated to the unscheduled maintenance event.

The three maintenance costs need to be known, they are: the Cost of Corrective Maintenance (CCM) the Cost of Preventive Maintenance (CPM) and the Total Cost of Maintenance (CTM). The CCM considers the cost of the equipment maintenance or replacement plus the working hours and the profit losses. The CPM considers only the cost of maintenance or equipment replacement and the equivalent working hours once the scheduled maintenance events were already planned in the energy production budget. The CCM for this analysed case was calculated in average as $\mathrm{R} \$ 2.330,00$ per event as the wind turbines are used to be stopped in average 12 hours until the analysed defect is solved. The profit losses related are about $\mathrm{R} \$ 1.230,00$ per event, considering the annual energy production average at the Wind Farm and the energy cost per MWh. The CPM is in average R\$ 1.100,00. After obtaining such information, Microsoft Excel is used again to calculate the probability of occurrences and the reliability of the system, based on the MTTF, scale and shape parameters already obtained before as shown below.

\begin{tabular}{|l|r|}
\hline \multicolumn{2}{|c|}{ Weibull Parameters } \\
\hline beta & 3,6879548 \\
\hline alfa & 7830,45014 \\
\hline \multicolumn{2}{|c|}{ Maintenance Costs } \\
\hline Scheduled & 1100 \\
\hline Unscheduled & 2115 \\
\hline
\end{tabular}

\begin{tabular}{|r|r|r|}
\hline MTTF $(\mathrm{h})$ & Probability & Reliability \\
\hline 4000 & $8,05 \%$ & $91,95 \%$ \\
\hline 4800 & $15,17 \%$ & $84,83 \%$ \\
\hline 5600 & $25,21 \%$ & $74,79 \%$ \\
\hline 6400 & $37,83 \%$ & $62,17 \%$ \\
\hline 7200 & $51,99 \%$ & $48,01 \%$ \\
\hline 8000 & $66,12 \%$ & $33,88 \%$ \\
\hline 8800 & $78,52 \%$ & $21,48 \%$ \\
\hline 9600 & $88,00 \%$ & $12,00 \%$ \\
\hline 10400 & $94,20 \%$ & $5,80 \%$ \\
\hline 11200 & $97,63 \%$ & $2,37 \%$ \\
\hline
\end{tabular}

Fig. 4. Results of Probability and Reliability based on Weibull parameters and maintenance costs

The results in the Figure 4 can be found by applying the equation 8 in the Microsoft Excel for the Probability of failure as equal to:

Weibull(MTTF; shape parameter; scale parameter; true) (8)

The equation for the Reliability is the complement of the failure Probability, as shown in the equation 9 as equal to:

1-Weibull(MTTF; shape parameter;scale parameter;true)(9)

The total cost of maintenance (CTM) for the scheduled and unscheduled maintenances, considering the probability and the reliability functions can be calculated with the equations (10) and (11) below: 


$$
\begin{aligned}
\mathrm{CTM}_{P} & =\frac{\mathrm{CPM} \cdot \mathrm{R}(\mathrm{t})+\mathrm{CCM} \cdot \mathrm{F}(\mathrm{t})}{\int_{0}^{\mathrm{t}} \mathrm{R}(\mathrm{s}) \mathrm{ds}} \\
\mathrm{CTM}_{\mathrm{R}} & =\frac{\mathrm{CPM} \cdot \mathrm{R}(\mathrm{t})+\mathrm{CCM} \cdot[1-\mathrm{R}(\mathrm{t})]}{\int_{0}^{\mathrm{t}} \mathrm{R}(\mathrm{s}) \mathrm{ds}}
\end{aligned}
$$

After calculating the Probability and Reliability and the total maintenance costs required by the method, all of the results must be expanded in several hypothetical intervals of MTTF, among the smallest to the biggest values of failure obtained in the historical records. The results can be plotted in order to conduct this analysis to the best scenario of maintenance, decreasing the maintenance costs and the risk of profit losses.

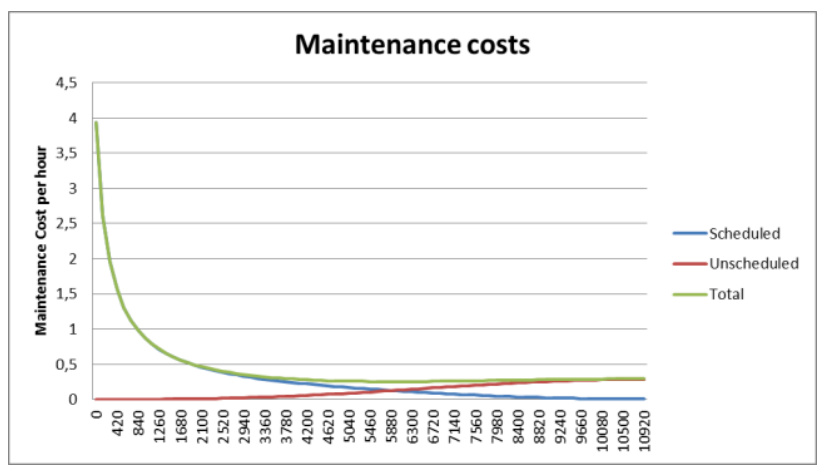

Fig. 5. Scheduled, Unscheduled and Total Costs of Maintenance

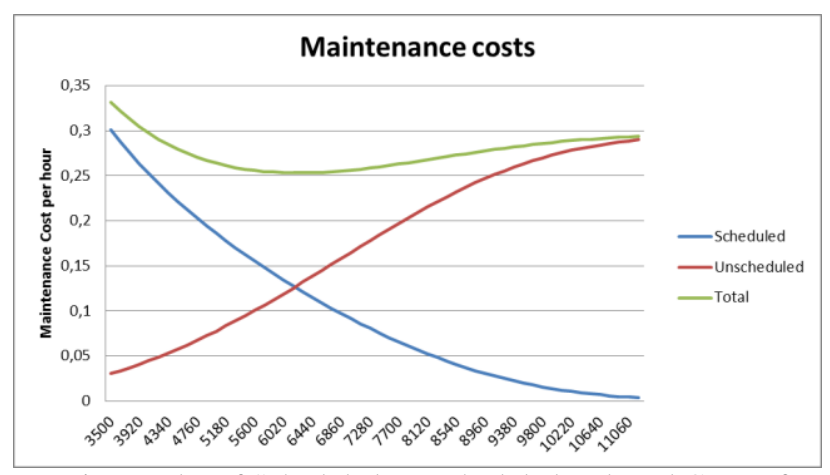

Fig. 6. Plot of Scheduled, Unscheduled and total Costs of Maintenance(zoom in)

The method determines that the best period of maintenance for the Wind Turbine and the Wind Farm assessed is that one in which the minimum value for the Total Cost of Maintenance per time unit can be found. Additionally, the mentioned result presents the optimized period for the maintenance. In the analysis above, the best scenario presents a maintenance cost per interval of hours about $\mathrm{R} \$$ 0,26 and the time interval to apply the maintenance about 5880 hours. It means that the scenario in which the best cost-benefit ratio can be seen is when the reliability is about $71 \%$ and the average total cost of maintenance (CTM) is $\mathrm{R} \$ 1.567,04$.

\section{Conclusion}

Currently, the maintenance schedules planned by the Suppliers consider only a preventive maintenance or a full replacement in any turbine equipment in the period of time previously established by contract in the beginning of the agreement negotiation. Due to, but not limited to this reason, most of the maintenance schedules are not improved by a Reliability approach. Part of this is due to the lack of awareness by the two parties, Owner and Supplier, of a simple Reliability analysis such as the one presented on this article in which the Wind Farm historical data and maintenance costs need to be respectively available and calculated. The gains in terms of revenue in the example shown in this article for a 20 year period, that refers to the Power Purchase Agreement for the assessed component is about $12 \%$ where can be conclude that the result totally justifies the application of the presented methodology. The method presented here can be applied not only for at the Wind Farms but also in several other areas where the MTTF and maintenance costs can be obtained.

\section{References}

[1] Institute of Electrical and Electronics Engineers (1990) IEEE Standard Computer Dictionary: A Compilation of IEEE Standard Computer Glossaries. New York, NY ISBN 1-55937-079-3

[2] Giorsetto, P., Utsurogi, K. F., Development of a New Procedure for Reliability Modeling of Wind Turbine Generators, IEEE Transactions on Power Apparatus and Systems, v. PAS-102, n. 1, pp 134-143, Jan. 1983

[3] Singh, C., Kim, Y., “An Efficient Technique for Reliability Analysis of Power Systems Including Time Dependent Sources", IEEE Transactions on Power Systems, v. 3, n. 3, pp. 1090-1096, Aug. 1988.

[4] Sayas, F. C., Allan, R. N., "Generation Availability Assessment of Wind Farms", IEE

Proc.-Gener. Transm. Distrib., v. 143, n. 5, Sep. 1996

[5] Roy Billinton, Power System Reliability Evaluation, Gordon and Breach Science Publishers, 1970, 299 pp

[6] W. Weibull: A statistical distribution function of wide applicability, J. Appl. Mech. 18, 293-296 (1951)

[7] Yunn-Kuang Chu and Jau-Chuan Ke , Computation approaches for Parameter Estimation of Weibull Distribution,

Mathematical and Computational Applications, 2012

Vol. 17, No. 1, pp. 39-47

[8] D. R. Dolas, M.D. Jaybhaye, S. D. Deshmukh, Estimation the System Reliability using Weibull Distribution, DOI: 10.7763/IPEDR. 2014. V75. 29 